\title{
Hysteroscopic removal of unabsorbed suture material causing "unexplained" menstrual disorder
}

\author{
Peter Hercz $\cdot$ Zsolt Makai
}

Received: 30 April 2010 /Accepted: 3 June 2010 / Published online: 7 August 2010

(C) Springer-Verlag 2010

Keywords Hysteroscopy · Unabsorbed suture material . Caesarean section

\section{Introduction}

In November 2005, a 33-year-old woman gravide 1 para 1 was admitted to our department in a gynecological outpatient clinic. Her symptoms included menometrorrhagia, lower abdominal pain, and pain experienced during intercourse. She also reported feeling 'unwell and distressed'. Her case history included a caesarean section in 1997. For several years her period had been heavy and regular, which later became irregular. After the caesarean section there were no signs of spotting or infection. Due to her complaints her sexual activity was very limited at the time and neither was she using contraception. In March 2005 she underwent a D\&C and diagnostic laparoscopy, which showed no pathological changes. In spite of the lack of pathology it was suggested she underwent a vaginal hysterectomy, which she refused.

Upon admission to our department in late 2005, subsequent examination by transvaginal sonography revealed no pathology and the uterine cavity looked normal as well. In order to establish the root of her symptoms, she

Pathological analysis/examination http://www.ncbi.nlm.nih.gov/pmc/ articles/PMC27273/?tool=pubmed display symptoms_ disappeared

P. Hercz $(\bowtie) \cdot$ Z. Makai

Department of Obstetrics and Gynecology,

Ferenc Jahn South-Pest Hospital of the Municipality of Budapest,

Köves u. 1,

Budapest 1204, Hungary

e-mail: drphercz@freemail.hu then underwent diagnostic hysteroscopy. The examination revealed several loops of suture material in the uterine cavity, which were deeply embedded in the endometrium on the upper part of the cavity. We planned to remove the suture material under intratracheal narcosis and a week later the patient was admitted again. The intrauterine pressure was $90 \mathrm{mmHg}$ and the flow rate $250 \mathrm{ml} / \mathrm{min}$. (Endomat, Storz Endoscopy Tuttlingen, Germany). The suture material was removed through Bettocchi Operating Sheath (Storz Endoscopy Tuttlingen, Germany). Firstly, it was cut with scissors in three locations, but then the use of biopsy forceps became necessary as it proved difficult to hold the material firm. The procedure lasted for $40 \mathrm{~min}$. Purisol solution $(9,800 \mathrm{ml})$ was washed into the uterine cavity with $9,000 \mathrm{ml}$ flowing back, meaning an $800-\mathrm{ml}$ deficit. There were no complications. Three months after the hysteroscopic removal of the suture material the patient began to experience normal menstrual cycles again and the low abdominal pain disappeared. She has been regularly attending our clinic every year since her operation. Her last visit was in July 2009, when she was found fit and well with her periods having returned to normal.

\section{Discussion}

Foreign bodies in the uterine cavity may change the endometrial architecture [2] and may cause menstrual disorders, infertility, low abdominal pain, purulent vaginal discharge, and sexual problems. Fragments of IUD, residual pregnancy products, fetal bones, gauze packing, and unabsorbable sutures have been found in the cavity in the uterus [2,3]. In our case unabsorbable suture material was discovered. Research has shown that, for example in 
rats, leucocystic infiltration was embedded in the uterine wall [4] after inserting nylon sutures into one of the uterine horns.

In our case the patient's complaints first appeared after her caesarean section in 1997 and increased throughout subsequent years. She was also distressed. Neither internal medical investigation nor vaginal sonography, D\&C or diagnostic laparoscopy could detect any pathology. Hysterectomy seemed to be the only solution, which the patient did not accept. It is most likely that during the caesarean section unabsorbable polyester suture was used, which slowly penetrated into the uterine cavity.

Other case studies also report on the same phenomenon. In two cases patients suffered from menstrual disorders following a caesarean section after which unabsorbable suture was found in the uterine cavity of both women [2]. One rather intriguing case was reported in 1950 [5]. A woman delivered babies by caesarean section both in 1930 and in 1936. Afterwards she experienced irregular uterine bleeding and purulent vaginal discharge. In 1938 an artificial menopause was induced with radium, but her period returned and she had persistent irregular uterine bleeding as well. In 1949 total hysterectomy was performed on her. In the uterine cavity silk suture was found in a small abscess.

It is unfortunate that in 1950 there was no practical possibility to treat the patient with hysteroscopy.
In the age of minimal access surgery, hysteroscopic removal of unabsorbed suture eliminated the complaints of a young woman and prevented an unnecessary hysterectomy.

\section{Conclusions}

Hysteroscopy may be the only tool which allows us to make a correct diagnosis and provide treatment in some obscure cases.

The authors have no commercial, proprietary, or financial interest in the products or companies described in this article. The authors have not received any financial support in any aspect of the work.

\section{References}

1. Bettochi S, Sardo AS, Pinto I., et al. (2009) Hysteroscopic removal of gauze packing inadvertenly sutured to the uterine cavity: report of 2 cases. J Minim Invas Gynec. 16:88-91

2. Xia EDA, Duan H, Huang X et al. (2003) Hysteroscopic removal of foreign bodies and it method of monitoring. Chinese Medical J. 116:125-128

3. Goldberg JM, Roberts S, (2008) Restoration of fertility after hysteroscopic removal of intrauterine bone fragments Obstet Gynec. 112:470-472

4. Havránek F, Dyková H, Tichy M, (1967) The effect of an intrauterine suture on fertility in the rat. J Reprod Fert. 14:15-19

5. Howkins J, (1950) Uterine sepsis from unabsorbable sutures. British Medical Journal 16:2-659 